\title{
Cytochrome P450 2A6 whole-gene deletion (CYP2A6*4) polymorphism reduces risk of lung cancer: A meta-analysis
}

\author{
Fadzrul H. Johani', Mohd S. A. Majid', Muhammad H. Azme', Azmawati M. Nawi'
}

\begin{abstract}
INTRODUCTION Lung cancer is the most commonly diagnosed cancer worldwide and is the leading cause of cancer death. Smoking is a major contributor to the pathogenesis of lung cancer. Cytochrome P450 2A6 (CYP2A6) is responsible for the metabolic activation of most tobacco carcinogens. CYP2A 6 genetic polymorphism can cause variations in the human metabolism of xenobiotics. We performed this meta-analysis to determine the association between whole-gene CYP2A6 deletion polymorphism $\left(C Y P 2 A 6^{*} 4\right)$ and lung cancer risk.

methods The PubMed, SAGE, Science Direct, the Cochrane Library and Ovid databases were searched for observational studies before October 2018. Methodological quality was assessed using the Newcastle-Ottawa Quality Assessment Scale (NOS).

RESULTS Nine case-control studies involving 4385 lung cancer cases and 4142 controls were included in the analysis. The random-effects model was used to combine results from individual studies. The pooled odds ratio was 0.39 (95\% CI: $0.27-0.56)$. There was no heterogeneity across studies $\left(\chi^{2}=2.49, \mathrm{p}=0.96, \mathrm{I}^{2}=0 \%\right)$. CONCLUSIONS Current evidence from the case-control studies suggests that the CYP2A6 whole-gene deletion polymorphism decreases the risk of lung cancer. Further research is needed to identify any potential confounding factors that may impact this association.
\end{abstract}

\author{
AFFILIATION \\ 1 Department of Community \\ Health, Faculty of Medicine, \\ University Kebangsaan \\ Malaysia, Cheras, Malaysia \\ CORRESPONDENCE TO \\ Azmawati M. Nawi. \\ Department of Community \\ Health, Faculty of Medicine, \\ University Kebangsaan \\ Malaysia, Jln Yaacob Latif, \\ Bdr Tun Razak, 56000, Cheras, \\ Kuala Lumpur, Malaysia. \\ E-mail: azmawati@ppukm. \\ ukm.edu.my \\ KEYWORDS \\ CYP2A6*4, Cytochrome P450 \\ 2A6, lung cancer, meta- \\ analysis, polymorphism
}

Received: 30 December 2019 Revised: 15 May 2020 Accepted: 15 May 2020

\section{INTRODUCTION}

In 2018, there were an estimated 18.1 million new cancer cases and 9.6 million cancer deaths, worldwide. Lung cancer is the most commonly diagnosed cancer worldwide, constituting $11.6 \%$ of total cancer cases, and is the leading cause of cancer death $(18.4 \%$ of total cancer deaths) ${ }^{1}$.

Smoking is a major contributor to the pathogenesis of lung cancer ${ }^{2}$. Even exposure to secondhand tobacco smoke increases the risk of developing lung cancer among non-smokers ${ }^{3,4}$. The pro-carcinogens specific to tobacco are clearly implicated in the progression of lung malignancies ${ }^{5,6}$. However, not all smokers will eventually develop lung cancer in their lifetime. These individual differences in lung cancer morbidity could be due to other determinants such as genetic susceptibility ${ }^{7,8}$ and environmental factors ${ }^{9-11}$. Thus, it is important to identify the genetic variants that influence the risk of lung cancer initiation ${ }^{12,13}$.

Most tobacco carcinogens require metabolic activation, which is mainly executed by the cytochrome P450 (CYP) enzymes ${ }^{14}$. Electrophile agents with short lifespans (which are produced in metabolic activation) cross-react with DNA, causing DNA damage and initiating tumours ${ }^{15,16}$. Among the CYP isozymes, CYP family 2 subfamily A member 6 (CYP2A6) is responsible for nicotine metabolism and for the metabolic activation of the tobacco-specific pro-carcinogens $N$-nitrosamine $N$-nitrosonornicotine (NNN) and 4-(methylnitrosoamino)-1-(3-pyridyl)-1butanone (NNK), which can eventually contribute to the progression of lung cancer ${ }^{5}$. 
Genetic polymorphism is defined as the inheritance of a trait controlled by a single genetic locus with two alleles in which the least common allele has a frequency of approximately $\geq 1 \%$ that can cause variation in the DNA sequence in individuals, groups, or populations ${ }^{17}$. The difference in DNA sequence may not alter the overall product sufficiently enough to produce a different protein but may affect the specific activity of the enzyme, and binding efficiencies such as those for transcription factors or membrane proteins, or other features and function ${ }^{18}$. Thus, Cytochrome P450 2A6 (CYP2A6) polymorphism can influence how humans metabolize xenobiotics.

Over the past two decades, several studies have assessed the association between CYP2A6 polymorphism, including whole-gene deletion of CYP2A6 on allele $4\left(C Y P 2 A 6^{*} 4\right)$, and the risk of lung cancer among different ethnic populations, but the results have been inconsistent ${ }^{12,19,20}$. Therefore, the present meta-analysis was performed to determine the association between $C Y P 2 A 6$ whole-gene deletion (CYP2A6*4) polymorphism and lung cancer risk.

\section{METHODS}

\section{Literature search}

This meta-analysis was performed based on the PRISMA (Preferred Reporting Items for Systematic Reviews and Meta-Analyses) statement ${ }^{21}$. A literature search was performed on five databases, namely PubMed, SAGE, ScienceDirect, the Cochrane Library, and Ovid. The search strategy utilized the PICO (Population, Intervention, Comparison, Outcome) framework to improve searching for clinical question ${ }^{22}$. The search terms used were: ['cytochrome P450 2A6' OR 'CYP2A6'] AND ['lung cancer' OR 'pulmonary cancer' OR 'respiratory cancer']. The search was not restricted to any duration or timeline.

\section{Study selection}

Two pairs of reviewers conducted the study selection in two phases after duplicate studies had been excluded. In the first phase, two reviewers (MSAM and MHA) independently screened the titles and abstracts of potential articles to be included in the study. During this phase, irrelevant studies were excluded, and a third reviewer (FHJ) resolved any disagreements. In the second phase, full-text articles were retrieved for detailed evaluation. Studies were included if they were published prior to October 2018 and met the following criteria: 1) observational study design, 2) presence of CYP2A6 whole-gene deletion (CYP2A6*4) polymorphism, and 3) presence of lung cancer. We excluded studies that were not published in English, reviews, case reports or animal studies, or if the full text was not available.

\section{Data extraction}

Articles that met the inclusion and exclusion criteria were retained for a full review. The characteristics of each study were examined and included study design, study location, type of population, sample size, sex, smoking status, matching criteria, genotyping method and genotype, and risk estimate value.

\section{Assessment of methodological quality}

Two authors assessed the quality of the selected articles independently, using the Newcastle-Ottawa Quality Assessment Scale (NOS) ${ }^{23}$ to examine for the concordance and average NOS score for each study. The NOS is widely used for quality assessment of observational studies ${ }^{24,25}$. It evaluates three components to quantify study quality, i.e. selection of study subjects, comparability of study groups and exposure or outcome ascertainment, which consists of eight items with a maximum score of 9 for each study. The scores of each item indicate the methodological quality of the study. A study is categorized as being of high, moderate or low quality, based on a total score of 7-9, 4-6 and 0-3, respectively ${ }^{26}$.

\section{Meta-analysis}

The random-effects mode ${ }^{27}$ was used to estimate the pooled effect size from the included studies. Odds ratio (OR) with 95\% confidence interval (CI) and a statistical measure of heterogeneity $\left(\mathrm{X}^{2}\right.$ and $\left.\mathrm{I}^{2}\right)$ were calculated using Review Manager 5.3 ${ }^{28}$. All selected studies were included in the meta-analysis. Subgroup and sensitivity analyses were performed if $\mathrm{p}<0.10$ and $\mathrm{I}^{2} \geq 50 \%$.

\section{RESULTS}

The search strategy returned a total of 172 articles. Initial screening excluded 35 articles due to duplication. Further screening of titles and abstracts excluded 41 irrelevant studies and nine review articles. The eligibility of the remaining 87 articles was assessed: 40 were excluded for studying 
different outcomes such as smoking behaviour, 28 were excluded for studying different exposures such as other CYP450 genotypes, eight were excluded as we were unable to extract raw data related to the whole deletion of allele $* 4 / * 4$, while two articles were excluded because we did not find data related to the whole deletion of allele $* 4 / * 4$ in the studies. The remaining nine articles ${ }^{12,20,29-35}$ were included in the meta-analysis. Figure 1 depicts the flow diagram describing article retrieval based on the PRISMA flow $\operatorname{diagram}^{21}$. The studies were carried out in Japan, China, Bangladesh, and Italy. Eight studies used Asian samples and only one study ${ }^{34}$ used Caucasian samples. Tables 1 and 2 present the characteristics of the included studies.

Two reviewers were independently assigned to score the NOS for each study individually. The correlation coefficient scores between the two authors were strong, with $r=0.91$ (Figure 2). Overall, the studies had moderate methodological quality as scored on NOS (mean score: 6.0; range: 2.0-7.5). Four studies were of high quality (NOS score: 7.0$9.0)^{20,29,32,34}$, four were of moderate quality (NOS score: 4.0-6.9 $)^{21,31,32,34}$, and only one study was of low quality (NOS score: $<4.0)^{35}$. The shape and symmetry of the funnel plot of $\log$ OR from the nine studies indicated that there was no publication bias (Figure $3)$. All studies had a high precision value.

Figure 4 shows the result of the meta-analysis using the random-effects model 28 , which combined the nine studies to explore the association between the CYP2A6* 4 polymorphism and the risk of lung cancer. The forest plot illustrates the spread of the studies' risk estimates and their CIs in relation to the pooled OR of meta-analysis. The pooled OR estimates showed that the $C Y P 2 A 6^{*} 4$ whole-gene deletion polymorphism significantly reduced the risk of lung cancer (pooled $\mathrm{OR}=0.39 ; 95 \%$ CI: 0.27-0.56). No heterogeneity was found across the studies (pheterogeneity $=0.96$, $\mathrm{I} 2=0 \%$ ). Subgroup analysis according to smoking status (Figure 5 ) showed that the pooled OR estimate of the CYP2A ${ }^{*} 4$ whole-gene deletion polymorphism remained significantly protective against lung cancer among 'All Smoker Status' (pooled OR $=0.41 ; 95 \%$ CI: 0.26-0.64), 'Mixed Smoking Status' (pooled OR=0.39; 95\% CI: 0.19-0.78) and 'Unknown Smoking Status'.

Figure 1. Flow chart of studies selection

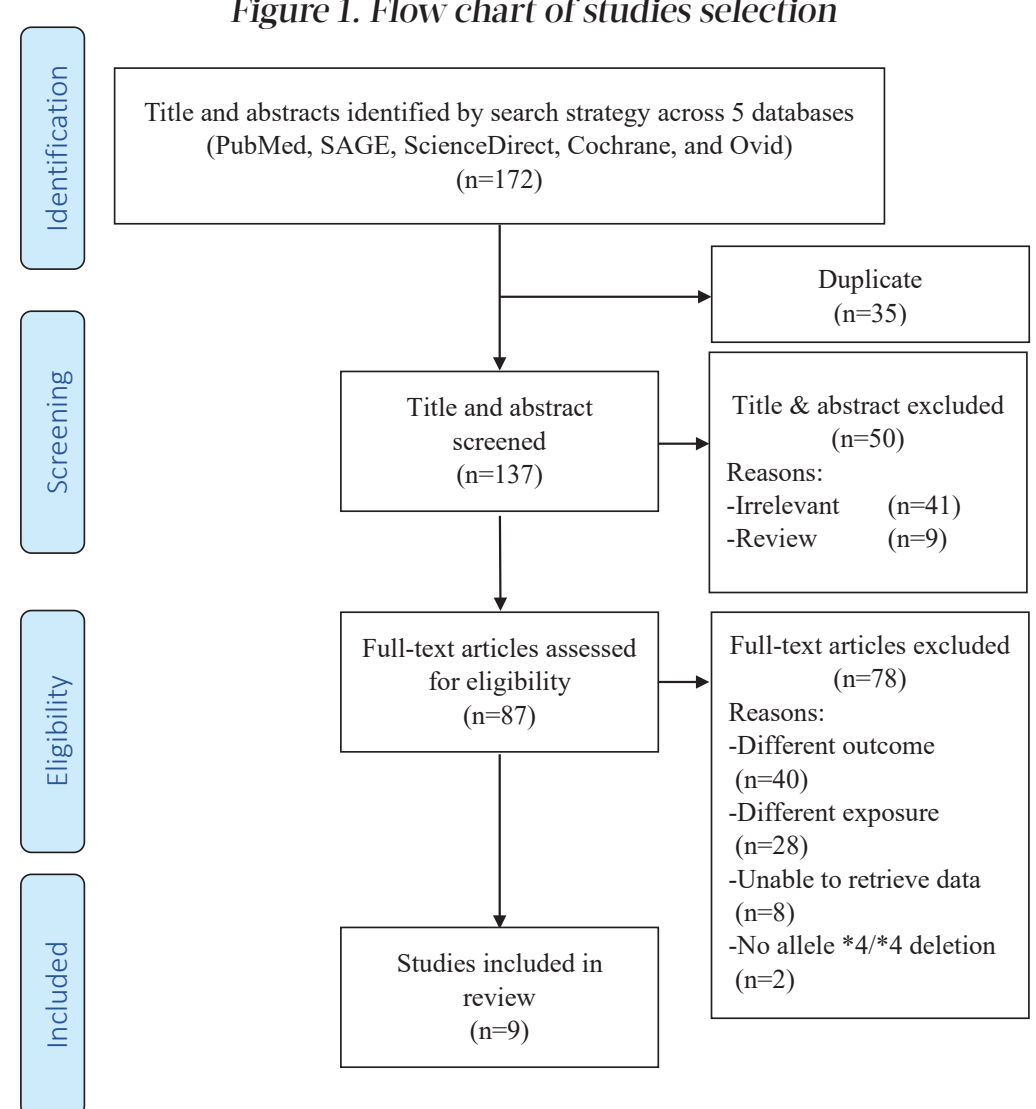


Table 1. Characteristics of studies included in the meta-analysis

\begin{tabular}{|c|c|c|c|c|c|c|c|c|}
\hline Study & $\begin{array}{c}\text { Year } \\
\text { Country \& } \\
\text { Population }\end{array}$ & $\begin{array}{l}\text { Study } \\
\text { design } \\
\text { (cases/ } \\
\text { controls) }\end{array}$ & $\begin{array}{l}\text { Crender; } \\
\text { Smoking } \\
\text { status }\end{array}$ & $\begin{array}{l}\text { Vatching } \\
\text { critcria }\end{array}$ & $\begin{array}{l}\text { Genotyping } \\
\text { methods }\end{array}$ & $\begin{array}{l}\text { Ilistological } \\
\text { type } \\
(\%)\end{array}$ & $\begin{array}{l}\text { CYP216 } \\
\text { genotype }\end{array}$ & Crude OR $\left(95^{\circ} \% \mathrm{CI}\right)$ \\
\hline $\begin{array}{l}\text { Hosono } \\
\text { et al. } .^{12}\end{array}$ & $\begin{array}{l}2015 \text { Japan } \\
\text { Asian }\end{array}$ & $\begin{array}{l}\text { Case- } \\
\text { control } \\
110 / 132\end{array}$ & $\begin{array}{l}\text { Both } \\
\text { All smoker }\end{array}$ & Age, sex & $\begin{array}{l}\text { PCR-Goodz } \\
\text { and Tyndale }\end{array}$ & SOCC (100) & $\begin{array}{l}\text { Group 1: } 1 / 1 \\
\text { Group 2: 1/7, 1/9, } \\
\text { 1/10, 1/13, 1/15, 8/9 } \\
\text { Group 3: } 1 / 4,1 / 41, \\
\text { 1/567C }>T, 7 / 9,7 / 11, \\
7 / 13,9 / 9,9 / 11 \\
\text { Group 4: 4/7, 4/9, } \\
4 / 13,4 / 18,7 / 567 C>T \\
\text { Group 5: 4/4, 4/5 }\end{array}$ & $\begin{array}{l}\text { Group 1: } 1 \text { (Ref.) } \\
\text { Group 2: } 1.00(0.49-2.07) \\
\text { Group 3: } 0.71(0.35-1.45) \\
\text { Group 4: } 0.13(0.04-0.45) \\
\text { Group 5: } 0.15(0.03-0.82)\end{array}$ \\
\hline $\begin{array}{l}\text { Islam } \\
\text { et al. }{ }^{29}\end{array}$ & $\begin{array}{l}2013 \\
\text { Bangladesh } \\
\text { Asian }\end{array}$ & $\begin{array}{l}\text { Case- } \\
\text { control } \\
106 / 116\end{array}$ & $\begin{array}{l}\text { Both } \\
\text { Mixed }\end{array}$ & $\begin{array}{l}\text { Age, sex, } \\
\text { smoking } \\
\text { status }\end{array}$ & PCR-RFLP & $\begin{array}{l}\text { SOCC (43.39) } \\
\text { AC }(34.91 \\
\text { SCC }(18.87) \\
\text { ASOC }(0.94)\end{array}$ & $\begin{array}{l}\text { Group 1: 1A/1A, } \\
\text { 1A/1B1, 1B1/1B1. } \\
\text { Group 2: } 1 \mathrm{~A} / 4 \\
\text { 1B1/4. 4/4 }\end{array}$ & $\begin{array}{l}\text { Group 1: } 1 \text { (Ref.) } \\
\text { Group 2: } 0.40(0.17-0.91)\end{array}$ \\
\hline $\begin{array}{l}\text { Tamaki } \\
\text { et al. } .^{30}\end{array}$ & $\begin{array}{l}2011 \text { Japan } \\
\text { Asian }\end{array}$ & $\begin{array}{l}\text { Case- } \\
\text { control } \\
192 / 203\end{array}$ & $\begin{array}{l}\text { Both } \\
\text { Mixed }\end{array}$ & Age, sex & PCR- Oscarson & $\begin{array}{l}\text { AC }(41.7) \\
\text { SOCC (24.5) } \\
\text { SCC (21.4) } \\
\text { ASOC (2.1) } \\
\text { LCC (0.5) } \\
\text { Unknown (3.6) }\end{array}$ & $\begin{array}{l}\text { Group 1: non-4/ } \\
\text { non-4 } \\
\text { Group 2: non-4/4 } \\
\text { Group 3: 4/4 }\end{array}$ & $\begin{array}{l}\text { Group 1: } 1 \text { (Ref.) } \\
\text { Group 2: } 0.92(0.60-1.42) \\
\text { Group 3: } 0.36(0.14-0.88)\end{array}$ \\
\hline $\begin{array}{l}\text { Rotunno } \\
\text { et al. }{ }^{34}\end{array}$ & $\begin{array}{l}2009 \text { Italy } \\
\text { Caucasian }\end{array}$ & $\begin{array}{l}\text { Case- } \\
\text { control } \\
1859 / 2019\end{array}$ & $\begin{array}{l}\text { Both } \\
\text { Mixed }\end{array}$ & $\begin{array}{l}\text { Age, sex, } \\
\text { area of } \\
\text { residence }\end{array}$ & SNP Assays & $\begin{array}{l}\text { AC (41) } \\
\text { SOCC (25.6) } \\
\text { SCC (10.2) } \\
\text { Other (21.5) } \\
\text { Unknown (1.8) }\end{array}$ & $\begin{array}{l}\text { Group 1: T/T } \\
\text { Group 2: T/A } \\
\text { Group 3: A/A }\end{array}$ & $\begin{array}{l}\text { Group 1: } 1 \text { (Ref.) } \\
\text { Group 2: } 0.74(0.55-1.00) \\
\text { Group 3: } 0.26(0.04-1.94)\end{array}$ \\
\hline $\begin{array}{l}\text { Fujieda } \\
\text { et al. }{ }^{31}\end{array}$ & $\begin{array}{l}2004 \text { Japan } \\
\text { Asian }\end{array}$ & $\begin{array}{l}\text { Case- } \\
\text { control } \\
1094 / 611\end{array}$ & $\begin{array}{l}\text { Both } \\
\text { All smokers }\end{array}$ & $\begin{array}{l}\text { No } \\
\text { matching }\end{array}$ & PCR-RFLP & $\begin{array}{l}\text { SOCC (26.9) } \\
\text { SCC (12.2) } \\
\text { AC (50.9) } \\
\text { Unknown } \\
(10.0)\end{array}$ & $\begin{array}{l}\text { Group 1: } 1 / 1 \\
\text { Group 2: } 1 / 4,1 / 7, \\
\text { 1/9, 1/10, 1/11 } \\
\text { Group } 3: 4 / 7,4 / 9, \\
4 / 10,4 / 11,7 / 7,7 / 9, \\
7 / 10,9 / 9,9 / 10,9 / 11 \text {, } \\
10 / 10 \\
\text { Group } 4: 4 / 4\end{array}$ & $\begin{array}{l}\text { Group 1: } 1 \text { (Ref.) } \\
\text { Group 2: } 0.59(0.44-0.79)^{\mathrm{a}} \\
\text { Group 3: } 0.52(0.37-0.72)^{\mathrm{a}} \\
\text { Group 4: } 0.30(0.16-0.57)^{\mathrm{a}}\end{array}$ \\
\hline $\begin{array}{l}\text { Ariyoshi } \\
\text { et al. }{ }^{32}\end{array}$ & $\begin{array}{l}2002 \text { Japan } \\
\text { Asian }\end{array}$ & $\begin{array}{l}\text { Case- } \\
\text { control } \\
370 / 380\end{array}$ & $\begin{array}{l}\text { Both } \\
\text { All smokers }\end{array}$ & $\begin{array}{l}\text { No } \\
\text { matching }\end{array}$ & PCR-Bell & $\begin{array}{l}\text { SCC (11.9) } \\
\text { SOCC }(28.4) \\
\text { AC }(52.1) \\
\text { Others }(7.6)\end{array}$ & $\begin{array}{l}\text { Group 1: } 1 \mathrm{~A} / 1 \mathrm{~A} \\
\text { Group 2: } 1 \mathrm{~A} / 1 \mathrm{~B} \\
\text { Group 3: } 1 \mathrm{~B} / 1 \mathrm{~B} \\
\text { Group 4: } 1 \mathrm{~A} / 4 \\
\text { Group 5: } 1 \mathrm{~B} / 4 \\
\text { Group 6: } 4 / 4\end{array}$ & $\begin{array}{l}\text { Group 1: } 1 \text { (Ref.) } \\
\text { Group 2: } 0.70(0.46-1.07) \\
\text { Group 3: } 0.60(0.37-0.99) \\
\text { Group 4: } 0.57(0.33-0.97) \\
\text { Group 5: } 0.56(0.34-0.92) \\
\text { Group 6: } 0.18(0.06-0.50)\end{array}$ \\
\hline $\begin{array}{l}\text { Tan } \\
\text { et al. }{ }^{20}\end{array}$ & $\begin{array}{l}2001 \text { China } \\
\text { Asian }\end{array}$ & $\begin{array}{l}\text { Case- } \\
\text { control } \\
151 / 326\end{array}$ & $\begin{array}{l}\text { Both } \\
\text { Mixed }\end{array}$ & Age, sex & PCR-0scarson & $\begin{array}{l}\operatorname{SCC}(58.3) \\
\text { AC }(31.1) \\
\text { Other (10.6) }\end{array}$ & $\begin{array}{l}\text { Group 1: } 1 / 1 \\
\text { Group 2: } 1 / 4,4 / 4\end{array}$ & $\begin{array}{l}\text { Group 1: } 1 \text { (Ref.) } \\
\text { Group 2: } 2.0(1.2-3.2)\end{array}$ \\
\hline $\begin{array}{l}\text { Miyamoto } \\
\text { et al. }{ }^{33}\end{array}$ & $\begin{array}{l}1999 \text { Japan } \\
\text { Asian }\end{array}$ & $\begin{array}{l}\text { Case- } \\
\text { control } \\
246 / 201\end{array}$ & $\begin{array}{l}\text { Both } \\
\text { NA }\end{array}$ & $\begin{array}{l}\text { No } \\
\text { matching }\end{array}$ & NA & NA & $\begin{array}{l}\text { Group 1: Wild/Wild } \\
\text { Group 2: Wild/Conv. } \\
\text { Group 3: Conv./Conv. } \\
\text { Group 4: Wild/Del. } \\
\text { Group 5: Conv./Del. } \\
\text { Group 6: Del./Del. }\end{array}$ & $\begin{array}{l}\text { Group 1: } 1 \text { (Ref.) } \\
\text { Group 2: } 0.59(0.34-1.02) \\
\text { Group 3: } 0.57(0.30-1.08) \\
\text { Group 4: } 0.29(0.14-0.59) \\
\text { Group 5: } 0.46(0.23-0.92) \\
\text { Group 6: } 0.25(0.08-0.83)\end{array}$ \\
\hline $\begin{array}{l}\text { Kamataki } \\
\text { et al. }{ }^{35}\end{array}$ & $\begin{array}{l}1999 \text { Japan } \\
\text { Asian }\end{array}$ & $\begin{array}{l}\text { Case- } \\
\text { control } \\
257 / 154\end{array}$ & $\begin{array}{l}\text { Both } \\
\text { NA }\end{array}$ & $\begin{array}{l}\text { No } \\
\text { matching }\end{array}$ & PCR-RFLP & NA & NA & NA \\
\hline
\end{tabular}

a Adjusted for age and smoking habit. PCR-RFLP: Polymerase Chain Reaction - Restriction Fragment Length Polymorphism. Del: Deletion-type, Conv: Conversion-type. SOCC: Squamous Cell Carcinoma. AC: Adenocarcinoma. SCC: Small Cell Carcinoma. ASOC: Adenosquamous Cell Carcinoma. LCC: Large Cell Carcinoma. 
Table 2. Genotype frequencies of CYP2A6*4 in studies included in the meta-analysis

\begin{tabular}{|c|c|c|c|c|c|c|c|}
\hline \multirow[t]{2}{*}{ Author } & \multirow[t]{2}{*}{ Year } & \multicolumn{3}{|c|}{ Case Genotype } & \multicolumn{3}{|c|}{ Control Genotype } \\
\hline & & $1 / * 1$ & -1/non-"1 & $\begin{array}{c}\text { non- } \\
-1 / \text { non- }\end{array}$ & $1 / 4$ & "1/non-"1 & $\begin{array}{c}\text { non- } 1 / 11011- \\
-1\end{array}$ \\
\hline Hosono et al. ${ }^{12}$ & 2015 & 2 & 32 & 76 & 8 & 48 & 76 \\
\hline Islam et al. ${ }^{29}$ & 2013 & 1 & 8 & 97 & 4 & 18 & 94 \\
\hline Tamaki et al..$^{30}$ & 2011 & 7 & 63 & 122 & 19 & 66 & 118 \\
\hline Rotunno et al. ${ }^{34}$ & 2009 & 2 & 101 & 1756 & 4 & 160 & 1855 \\
\hline Fujieda et al. ${ }^{31}$ & 2004 & 25 & 301 & 768 & 28 & 186 & 397 \\
\hline Ariyoshi et al..$^{32}$ & 2002 & 5 & 98 & 267 & 19 & 117 & 244 \\
\hline Tan et al..$^{20}$ & 2001 & 1 & 38 & 112 & 5 & 46 & 275 \\
\hline Miyamoto et al..$^{33}$ & 1999 & 5 & 48 & 193 & 9 & 60 & 132 \\
\hline Kamataki et al..$^{35}$ & 1999 & 2 & - & 255 & 6 & - & 148 \\
\hline
\end{tabular}

Figure 2. The research quality assessment of each study was assessed using NOS with two independent reviewers.

(Pooled OR=0.34; 95\% Cl: 0.14-0.85)
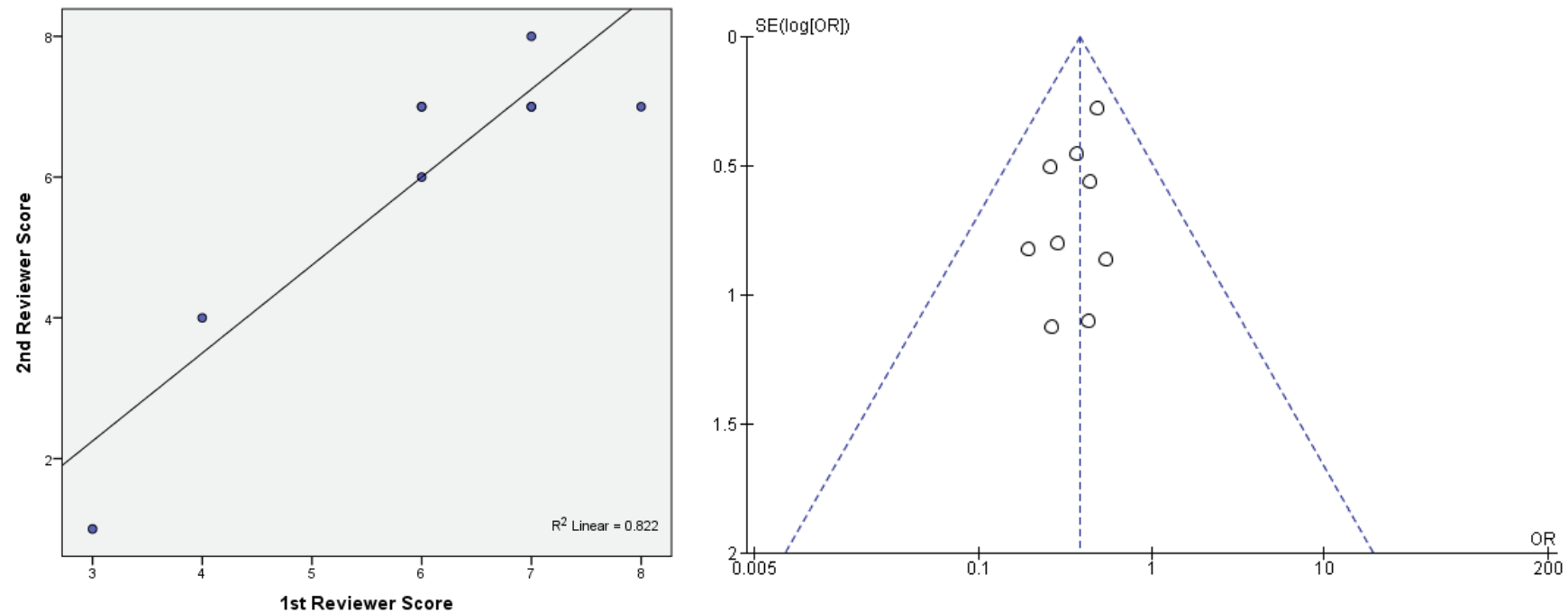

Figure 4. Forest plot analysis

\begin{tabular}{|c|c|c|c|c|c|c|c|c|c|c|c|}
\hline \multirow[b]{2}{*}{ Study or Subgroup } & \multicolumn{2}{|c|}{ Lung Cancer } & \multicolumn{2}{|c|}{ Non-Lung Cancer } & \multicolumn{3}{|c|}{ Odds Ratio } & \multirow{2}{*}{\multicolumn{4}{|c|}{$\begin{array}{c}\text { Odds Ratio } \\
\text { M-H, Random, } 95 \% \mathrm{Cl}\end{array}$}} \\
\hline & Events & Total & Events & Total & Weight & M-H, Random, $95 \% \mathrm{Cl}$ & Year & & & & \\
\hline Miyamoto et al. 1999 & 5 & 246 & 9 & 201 & $10.2 \%$ & $0.44[0.15,1.34]$ & 1999 & & & D. & \\
\hline Kamataki et al. 1999 & 2 & 257 & 6 & 154 & $4.8 \%$ & $0.19[0.04,0.97]$ & 1999 & & & & \\
\hline Tan et al. 2001 & 1 & 151 & 5 & 326 & $2.7 \%$ & $0.43[0.05,3.70]$ & 2001 & & & & \\
\hline Ariyoshi et al. 2002 & 5 & 370 & 19 & 380 & $12.7 \%$ & $0.26[0.10,0.70]$ & 2002 & & & & \\
\hline Fujieda et al. 2004 & 25 & 1094 & 28 & 611 & $41.7 \%$ & $0.49[0.28,0.84]$ & 2004 & & $\rightarrow-$ & & \\
\hline Rotunno et al. 2009 & 2 & 1859 & 4 & 2019 & $4.4 \%$ & $0.54[0.10,2.97]$ & 2009 & & & & \\
\hline Tamaki et al. 2011 & 7 & 192 & 19 & 203 & $15.8 \%$ & $0.37[0.15,0.89]$ & 2011 & & & & \\
\hline Islam et al. 2013 & 1 & 106 & 4 & 116 & $2.6 \%$ & $0.27[0.03,2.42]$ & 2013 & & & & \\
\hline Hosono et al. 2015 & 2 & 110 & 8 & 132 & $5.1 \%$ & $0.29[0.06,1.38]$ & 2015 & & & & \\
\hline Total $(95 \% \mathrm{Cl})$ & & 4385 & & 4142 & $100.0 \%$ & $0.39[0.27,0.56]$ & & & & & \\
\hline Total events & 50 & & 102 & & & & & & & & \\
\hline $\begin{array}{l}\text { Heterogeneity: } \operatorname{Tau}^{2}= \\
\text { Test for overall effect: }\end{array}$ & $\begin{array}{l}.00 ; \mathrm{Chi}^{2} \\
=5.20(\mathrm{P}\end{array}$ & $\begin{aligned} & 2.49,0 \\
= & 0.000\end{aligned}$ & $\begin{array}{l}\mathrm{ff}=8(P=0 \\
01)\end{array}$ & $;\left.\right|^{2}=0$ & & & & $\stackrel{t}{0.005}$ & 0.1 & 10 & 200 \\
\hline
\end{tabular}


Figure 5. Subgroup analysis according to smoking status

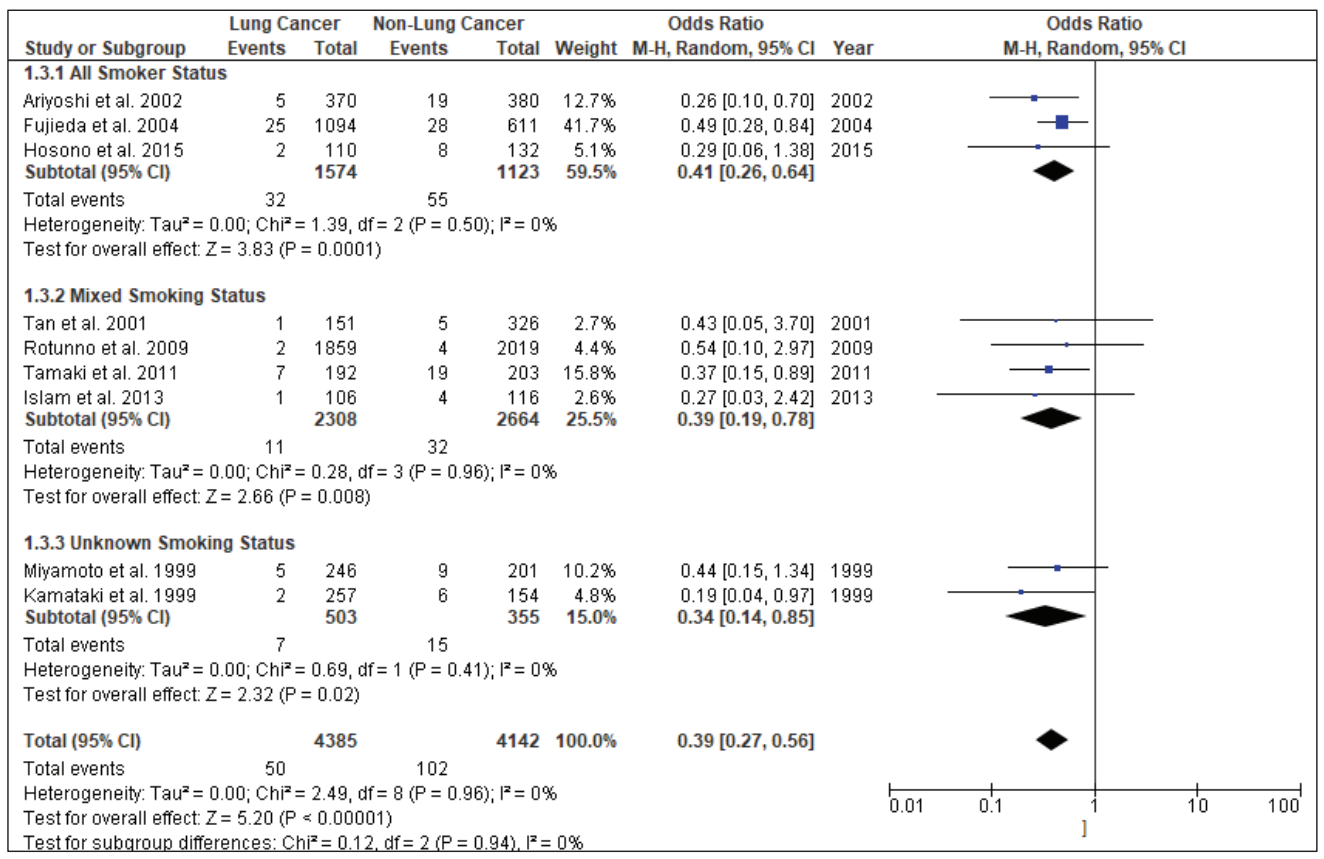

\section{DISCUSSION}

A comprehensive search of different databases was used to yield the most relevant results and incorporated the available epidemiologic evidence to explore the relationship between the CYP2A6*4 whole-gene deletion polymorphism and the risk of lung cancer. Nine case-control studies fulfilled the criteria addressing this issue. The analysis, involving 4385 lung cancer cases and 4142 controls, suggested that $C Y P 2 A 6$ polymorphism significantly reduces the risk of lung cancer ( pooled OR $=0.39 ; 95 \%$ CI: 0.27-0.56), with homogeneity observed across studies $\left(X^{2}=2.49, \mathrm{p}=0.96, \mathrm{I}^{2}=0 \%\right)$. Thus, subgroup and sensitivity analyses were not explored.

There are two possible explanations for the finding of relative risk reduction as revealed by the analysis. First, CYP2A6 is mainly found in the liver and other tissues such as the nasal epithelium, trachea, lung, and oesophagus $^{36,37}$. CYP2A6 metabolizes a few but specific xenobiotics that include nicotine and some tobacco specific nitrosamines that enter the human body. Metabolic activation by CYP2A6 enzymes generally produces a short-lived electrophile agent that reacts with DNA, causing DNA damage and inducing a tumour $^{16}$. In tobacco smoke, CYP2A6 is the main enzyme activating the tobacco-specific $N$-nitrosamines
NNK and NNN, which are pro-carcinogens ${ }^{5,6}$. Thus, in individuals with the inactive $C Y P 2 A 6$ genotype, the CYP2A6 enzyme might not affect metabolic activation of N-nitrosamines and subsequently reduce the risk of lung cancer. Second, CYP2A6 is a major enzyme responsible for nicotine metabolism ${ }^{38}$, where inactive CYP2A6 causes lower nicotine dependence and thus affects smoking behaviour ${ }^{39-41}$.

Of the included studies, only three studies ${ }^{12,31,32}$ recruited only smokers as participants; the remaining studies had mixed populations of smokers and neversmokers. The mixed-smoker status studies yielded non-significant findings or smaller risk estimates, which may be explained by the fact that the probability of lung cancer occurrence may be similar among never-smokers with different CYP2A6 genotypes, where the resulting phenotype is not expressed in people who do not smoke. Thus, when the original studies included both smokers and non-smokers, the association between the CYP2A6 polymorphism genotype and risk of lung cancer may have been attenuated. This may explain why some of the studies did not find any significant association ${ }^{19,20,29,42}$.

Besides that, cigarette smoking is strongly associated with squamous cell carcinoma compared to adenocarcinoma ${ }^{43-45}$. However, in the present meta- 
analysis, all but one study included different lung cancer histology types; Hosono et al. ${ }^{12}$ only recruited squamous cell carcinoma cases.. The study by Islam et al. ${ }^{29}$ had the highest proportion of squamous cell carcinoma cases $(43.39 \%)$, while the other studies had 24.5-28.4\% squamous cell carcinoma cases ${ }^{30-32,34}$. These differences in the proportion of histological types might explain the discrepancy of the findings among the studies on CYP2A6 polymorphism and lung cancer risk.

\section{Limitations}

The present meta-analysis findings should be interpreted with caution. Six studies did not stratify the smoking status to assess the association between CYP2A6 polymorphism and lung cancer. Therefore, the true relationship between $C Y P 2 A 6$ polymorphism and risk of lung cancer in current-smokers and neversmokers could not be tested in these six studies. In addition, lung cancer might arise due to occupational carcinogen exposure, such as organic dust and silica dust ${ }^{46,47}$, which may confound the association between CYP2A6 polymorphism and lung cancer risk. However, none of the included studies adjusted for occupational carcinogen hazard exposure originally. The relationship may be also affected by differences in the demographic characteristics and socioeconomic class of the respondents ${ }^{48}$, which some of the studies did not mention.

\section{CONCLUSIONS}

The evidence from the case-control studies included in the present meta-analysis shows that people with CYP2A6* 4 whole-gene deletion have a decreased risk of lung cancer. Further research is needed to identify any potential confounding factors that may impact this association.

\section{REFERENCES}

1. Bray F, Ferlay J, Soerjomataram I, Siegel RL, Torre LA, Jemal A. Global cancer statistics 2018: GLOBOCAN estimates of incidence and mortality worldwide for 36 cancers in 185 countries. CA Cancer J Clin. 2018;68:394424. doi:10.3322/caac.21492

2. World Health Organization, International Agency for Research on Cancer. Tobacco smoke and involuntary smoking. Volume 83 of IARC Monographs on the Evaluation of Carcinogenic Risks to Humans. Lyon, France: International Agency for Research on Cancer;
2004. https://monographs.iarc.fr/wp-content/ uploads/2018/06/mono83.pdf. Accessed December 30, 2019.

3. Zhong L, Goldberg MS, Parent MÉ, Hanley JA. Exposure to environmental tobacco smoke and the risk of lung cancer: a meta-analysis. Lung Cancer. 2000;27:3-18. doi:10.1016/s0169-5002(99)00093-8

4. Taylor R, Gumming R, Woodward A, Black M. Passive smoking and lung cancer: a cumulative metaanalysis. Aust N Z J Public Health. 2001;25:203-211. doi:10.1111/j.1467-842x.2001.tb00564.x

5. Crespi CL, Penman BW, Gelboin HV, Gonzalez FJ. A tobacco smoke-derived nitrosamine, 4-(methytnitrosamino)-1(3-pyridyl)-1-butanone, is activated by multiple human cytocbrome P450s including the polymorphic human cytochrome P4502D6. Carcinogenesis. 1991;12:11971201. doi:10.1093/carcin/12.7.1197

6. Hecht SS, Chen CHB, Hoffmann D. A study of tobacco carcinogenesis. 17. Tobacco-specific nitrosamines: occurrence, formation, carcinogenicity, and metabolism. Acc Chem Res. 1979;12:92-98. doi:10.1021/ar50135a003

7. Spitz MR, Wei Q, Dong Q, Amos CI, Wu X. Genetic susceptibility to lung cancer: the role of DNA damage and repair. Cancer Epidemiol Biomarkers Prev. 2003;12:689698. PMID:12917198

8. Matakidou A, Eisen T, Houlston R. Systematic review of the relationship between family history and lung cancer risk. Br J Cancer. 2005;93:825. doi:10.1038/sj.bjc.6602769

9. Zhao Y, Wang S, Aunan K, Seip HM, Hao J. Air pollution and lung cancer risks in China-a meta-analysis. Sci Total Environ. 2006;366:500-513. doi:10.1016/j.scitotenv.2005.10.010

10. Hamra GB, Guha N, Cohen A, et al. Outdoor particulate matter exposure and lung cancer: a systematic review and meta-analysis. Environ Health Perspect. 2014;122(9). doi:10.1289/ehp/1408092

11. Celik I, Gallicchio L, Boyd K, et al. Arsenic in drinking water and lung cancer: a systematic review. Environ Res. 2008;108:48-55. doi:10.1016/j.envres.2008.04.001

12. Hosono H, Kumondai M, Arai T, et al. CYP2A6 genetic polymorphism is associated with decreased susceptibility to squamous cell lung cancer in Japanese smokers. Drug Metab Pharmacokinet. 2015;30:263-268. doi:10.1016/j.dmpk.2015.04.002.

13. Goode EL, Ulrich GM, Potter JD. Polymorphisms in DNA repair genes and associations with cancer risk. Cancer Epidemiol Biomarkers Prev. 2002;11:1513-1530. PMID:12496039

14. Yamazaki H, Inui Y, Yun CH, Guengerich FP, Shimada T. Cytochrome P450 2E1 and 2A6 enzymes as major catalysts for metabolic activation of $\mathrm{N}$-nitrosodialkylamines and tobacco-related nitrosamines in human liver microsomes. Carcinogenesis. 1992;13:1789-1794. doi:10.1093/carcin/13.10.1789

15. Guengerich FP, Shimada T. Activation of procarcinogens by human cytochrome P450 enzymes. Mutat Res. 
1998;400:201-213. doi:10.1016/s0027-5107(98)00037-2

16. Nakajima M, Yamamoto T, Nunoya K, et al. Role of human cytochrome P4502A6 in C-oxidation of nicotine. Drug Metab Dispos. 1996;24:1212-1217.

17. Ismail S, Essawi M. Genetic polymorphism studies in humans. Middle East Journal of Medical Genetics. 2012;1:57-63. doi:10.1097/01.mxe.0000415225.85003.47

18. Phillips T. Genetic Polymorphism-Different Does Not Mean Mutated: Multiple Forms of a Single Gene. https:// www.thebalance.com/genetic-polymorphism-what-isit-375594. Accessed December 18, 2018.

19. Loriot MA, Rebuissou S, Oscarson M, et al. Genetic polymorphisms of cytochrome P450 2A6 in a case-control study on lung cancer in a French population. Pharmacogenetics. 2001;11:39-44. doi: 10.1097/00008571-200102000-00005

20. Tan W, Chen GF, Xing DY, Song CY, Kadlubar FF, Lin DX. Frequency of $C Y P 2 A 6$ gene deletion and its relation to risk of lung and esophageal cancer in the Chinese population. Int J Cancer. 2001;95:96-101. doi:10.1002/10970215(20010320)95:2<96::aid-ijc1017>3.0.co;2-2

21. Moher D, Liberati A, Tetzlaff J, Altman DG, PRISMA Group. Preferred reporting items for systematic reviews and meta-analyses: the PRISMA statement. Ann Intern Med. 2009;151:264-269. doi:10.7326/0003-4819-151-4-200908180-00135

22. Schardt C, Adams MB, Owens T, Keitz S, Fontelo P. Utilization of the PICO framework to improve searching PubMed for clinical questions. BMC Med Inform Decis Mak. 2007;7:16. doi:10.1186/1472-6947-7-16

23. Wells G, Shea B, O'Connell D, et al. The Newcastle-Ottawa Scale (NOS) for assessing the quality of nonrandomized studies in meta-analyses. http://www.ohri.ca/programs/ clinical_epidemiology/oxford.asp. Published 2012. Accessed December, 2018.

24. Stang A. Critical evaluation of the Newcastle-Ottawa scale for the assessment of the quality of nonrandomized studies in meta-analyses. Eur J Epidemiol. 2010;25:603605. doi:10.1007/s10654-010-9491-z

25. Modesti PA, Reboldi G, Cappuccio FP, et al. Panethnic differences in blood pressure in Europe: a systematic review and meta-analysis. PLoS One. 2016;11:e0147601. doi:10.1371/journal.pone.0147601

26. Tang S, Wang Y, Gong X, Wang G. A meta-analysis of maternal smoking during pregnancy and autism spectrum disorder risk in offspring. Int J Environ Res Public Health. 2015;12:10418-10431. doi:10.3390/ijerph120910418

27. Borenstein M, Hedges LV, Higgins JP, Rothstein HR. A basic introduction to fixed-effect and random-effects models for meta-analysis. Res Synth Methods. 2010;1:97111. doi:10.1002/jrsm.12

28. Review Manager [computer program]. Version 5.3. Copenhagen: The Nordic Cochrane Centre, The Cochrane Collaboration, 2014.

29. Islam MS, Ahmed MU, Sayeed MSB, et al. Lung cancer risk in relation to nicotinic acetylcholine receptor, $C Y P 2 A 6$ and CYP1A1 genotypes in the Bangladeshi population. Clin Chim Acta. 2013;416:11-19. doi:10.1016/j.cca.2012.11.011

30. Tamaki Y, Arai T, Sugimura H, et al. Association between cancer risk and drug-metabolizing enzyme gene (CYP2A6, CYP2A13, CYP4B1, SULT1A1, GSTM1, and GSTT1) polymorphisms in cases of lung cancer in Japan. Drug Metab Pharmacokinet. 2011;26:516-522. doi:10.2133/dmpk.dmpk-11-rg-046

31. Fujieda M, Yamazaki H, Saito T, et al. Evaluation of CYP2A6 genetic polymorphisms as determinants of smoking behavior and tobacco-related lung cancer risk in male Japanese smokers. Carcinogenesis. 2004;25:24512458. doi:10.1093/carcin/bgh258

32. Ariyoshi N, Miyamoto M, Umetsu Y, et al. Genetic polymorphism of CYP2A6 gene and tobacco-induced lung cancer risk in male smokers. Cancer Epidemiol Biomarkers Prev. 2002;11:890-894. PMID:12223434

33. Miyamoto M, Umetsu Y, Dosaka-Akita H, et al. CYP2A6 gene deletion reduces susceptibility to lung cancer. Biochem Biophys Res Commun. 1999;261:658-660. doi:10.1006/bbrc.1999.1089

34. Rotunno M, Yu K, Lubin JH, et al. Phase I metabolic genes and risk of lung cancer: multiple polymorphisms and mRNA expression. PloS One. 2009;4:e5652. doi:10.1371/journal.pone.0005652

35. Kamataki T, Nunoya K, Sakai Y, Kushida H, Fujita K. Genetic polymorphism of CYP2A6 in relation to cancer. Mutat Res. 1999;428:125-130. doi:10.1016/s1383-5742(99)00040-x

36. Koskela S, Hakkola J, Hukkanen J, et al. Expression of CYP2A genes in human liver and extrahepatic tissues. Biochem Pharmacol. 1999;57:1407-1413. doi:10.1016/s0006-2952(99)00015-5

37. Zanger UM, Schwab M. Cytochrome P450 enzymes in drug metabolism: regulation of gene expression, enzyme activities, and impact of genetic variation. Pharmacol Ther. 2013;138:103-141. doi:10.1016/j.pharmthera.2012.12.007

38. Messina E, Tyndale R, Sellers E. A major role for CYP2A6 in nicotine C-oxidation by human liver microsomes. J Pharmacol Exp Ther. 1997;282:1608-1614.

39. Tanner JA, Zhu AZ, Claw KG, et al. Novel CYP2A6 diplotypes identified through next-generation sequencing are associated with in-vitro and in-vivo nicotine metabolism. Pharmacogenet Genomics. 2018;28:7-16. doi:10.1097/fpc.0000000000000317

40. Olfson E, Bloom J, Bertelsen S, et al. CYP2A6 metabolism in the development of smoking behaviors in young adults. Addict Biol. 2018;23:437-447. doi:10.1111/adb.12477

41. Tyndale RF, Sellers EM. Variable CYP2A6-mediated nicotine metabolism alters smoking behavior and risk. Drug Metab Dispos. 2001;29:548-552. PMID:11259349

42. Huang FM, Chen HC, Khan MA, et al. CYP2A6, CYP1A1, and CYP2D6 polymorphisms in lung cancer patients from Central South China. Med Oncol. 2013;30:521. doi:10.1007/s12032-013-0521-z 
43. Khuder SA. Effect of cigarette smoking on major histological types of lung cancer: a meta-analysis. Lung Cancer. 2001;31:139-148. doi:10.1016/s0169-5002(00)00181-1

44. Risch HA, Howe GR, Jain M, Burch JD, Holowaty EJ, Miller AB. Are female smokers at higher risk for lung cancer than male smokers? A case-control analysis by histologic type. Am J Epidemiol. 1993;138:281-293. doi:10.1093/oxfordjournals.aje.a116857

45. Barbone F, Bovenzi M, Cavallieri F, Stanta G. Cigarette smoking and histologic type of lung cancer in men. Chest. 1997;112:1474-1479. doi:10.1378/chest.112.6.1474

46. Peters S, Kromhout H, Olsson AC, et al. Occupational exposure to organic dust increases lung cancer risk in the general population. Thorax. 2012;67:111-116. doi:10.1136/thoraxjnl-2011-200716

47. Pelucchi C, Pira E, Piolatto G, Coggiola M, Carta P, La Vecchia C. Occupational silica exposure and lung cancer risk: a review of epidemiological studies 1996-2005. Ann Oncol. 2006;17:1039-1050. doi:10.1093/annonc/mdj125

48. Klassen AC, Hsieh S, Pankiewicz A, Kabbe A, Hayes J, Curriero F. The association of neighborhood-level social class and tobacco consumption with adverse lung cancer characteristics in Maryland. Tob Induc Dis. 2019;17(January). doi:10.18332/tid/100525
CONFLICTS OF INTEREST

The authors have completed and submitted the ICMJE Form for Disclosure of Potential Conflicts of Interest and none was reported.

\section{FUNDING}

There was no source of funding for this research.

\section{AUTHORS' CONTRIBUTIONS}

AMN initiated the idea and also designed the current study. FHJ and MSAM, simultaneously performed independent searches and also conducted the subjective as well as the objective quality assessment of the retrieved articles. MHA screened the titles and abstracts of the retrieved articles to identify the relevance of the reports to the objective of this review using the eligibility criteria. All four authors contributed in the final synthesis and writing of the manuscript. FHJ and MSAM performed the meta-analysis for this study.

PROVENANCE AND PEER REVIEW

Not commissioned; externally peer reviewed. 